\title{
STUDY ON THE IMPORTANCE OF LAND-CENTERED TRAINING FOR THE FREESTYLE SWIMMING TECHNIQUE FOR MILITARY STUDENTS
}

\author{
Fabiana MARTINESCU-BĂDĂLAN \\ “Nicolae Bălcescu” Land Forces Academy, Sibiu, Romania \\ martinescu_fabiana@yahoo.com
}

\begin{abstract}
Swimming is a very important component of the military training. This can be proven by the fact that swimming is a beneficial sport for the human body because the joints are not tensed like in running, increases heart rate, but also stimulates blood circulation. At the same time, while swimming, absolutely all muscle groups are involved, which means automatically shaping them and, over time, increasing the body's resistance.

The study found the following:

- students' results at the $50 \mathrm{~m}$ freestyle swimming test are positively influenced if an additional training program is respected;

- additional physical training leads to improved performance.
\end{abstract}

Keywords: swimming, freestyle, practice, to improve

\section{Introduction}

"Swimming is different (...) It is what makes it so easy to leave a comfortable bed right before sunrise on a frozen morning or on a choking summer day just to get to the pool on time"[1].

In the case of the Land Forces Academy students, swimming can be a relaxing and restful activity after demanding courses or military training and, at the same time, a way of developing the physical fitness of students. Another argument to swim is that it increases flexibility [2] because this activity improves mobility better than any other exercise on land. "Since swimming is not a fountain of cardiologic youth, a 1988 study of cardiologists and physiologists at the University of Texas Dallas Health and Science Center showed that adults who did not do regular physical activity, have improved heart function significantly, only within three months of starting a swimming program.

Heart beats are slower and stronger, and blood circulation is performed more efficiently. Swimmers are renowned as having lower blood pressure, slower pulse, and greater physical activity tolerance than other people of the same age. Above all, it is a benefit of aerobic capacity that a mile in the aquatic environment is equal to four miles of running"'[1].

\section{Research objectives}

Identifying the desire of military students to practice swimming outside of military physical education classes;

Student availability to follow the additional training program;

Improving the military effort of the students. 


\section{Sample of research}

The research was based on two samples of students from the "Nicolae Bălcescu" Land Forces Academy in Sibiu, the first sample being composed of 30 students (20 years \pm 1 ) who were part of a trial group and the second - a number of 30 students (20 years \pm 1 ), but who represented the experimental group.

The difference between these two groups is that the students in the experimental group have additionally participated in a landbased training program (in the fitness gym) respecting a centralized program, while the students in the trial group participated only in the military physical education classes established in their curriculum.

\section{The freestyle swimming technique}

The freestyle swimming technique is characterized by alternating vertical movement of the lower limbs, the cyclic movement of the upper limbs, but also their simultaneous coordination.

Upper limb movement is done in two distinct levels:

a) air;

b) aquatic rafting.

a) The air travel made with the arms or the recovery phase starts when the shoulder comes out of the water. „The maximum point of flexion will be when the palm and elbow reach the shoulder, a motion which becomes easier by the gradual twisting of the shoulder axis. After crossing the shoulder line, the elbow begins the extension and the arm will rotate internally so that the palm orientation is outward, ensuring a water entry with a minimum of turbulence. Avoiding side pendulum scan be done by an upright lifting of the elbow as close to the shoulder"'[3].

Rowing is a fairly complex process, which is why it goes through several stages.

The first stage begins by inserting one of the arms with the tip of the fingers in the water, the elbow flexing slightly and the palm in the prone (the movement of the rotation of the forearm, the palm passing inward).The order of entry into the water is the following: fingers, palms, forearms and arms that must penetrate through the same spot by hitting the water with the fingers.

The second stage is performed starting with the arm stretching under the horizontal of the water, followed by the extension of the elbow and the rotation of the arm until the palm is downward.,,No force will be applied to the arm after it has entered the water to maintain the propulsion efficiency provided by the opposite arm that is in the middle of the underwater path. The velocity should decrease from the moment of water entry to full extension, pushed forward by the body until near the end of the propulsive moment of the other arm. "'[4]

The third stage continues the pulling process by twisting the palm to the outside, and when the palm is lowered, the water grip is executed, the moment the elbow reaches it. After their execution, traction will take place until the palm and elbow reach the shoulder (on the same line), the arm being positioned at an angle of $90^{\circ}$ to the body. In traction, ,lowering the arm prevents sinking of the face during the inspiration during the position of the opposite arm in the air. Holding and lowering the arm positions the arm to start the first part of the underwater propulsive stage. Inward movement is depicted on a semicircular trajectory and the palm travels downward, inwardly and upwards until it reaches the midline. In the traction phase, the palm will slowly rotate inward, so at the end it is oriented inward and upward. The rotational speed of the hand from the outside to the inside is the key to traction efficiency"'[4].

It is imperative that the swimmer coordinates the upper limbs so that the arms alternate into the water, more precisely, when one arm enters the water, the other end the traction. ,Another important aspect of arm co-ordination is to keep the front arm stretched (without letting it sink) until the arm that is pulling completes the push, so the swimmer's body slides in a straight line during the push. However, this will lead to a deceleration of the front arm traction, but perhaps more likely to reduce 
the propulsive force that would occur if an extended part of the front arm would be pushed forward against the water during the pushing of the arm that is pulling. The deceleration recorded at end of the push and the start of the traction is offset by the propulsion from the traction "[4].

The co-ordination of breathing with the movement of the arms is done in two distinct phases, namely inspiration and exhalation. When the arm is pulled out of the water and the air inhalation begins, inspiration is made, and the exhalation is made under water when the other arm finishes the traction and begins to push, ending when the face approaches the surface of the water to perform a new inspiration. ,Inspiration takes place on the first side of the airway of the arm, and on the second stage the face returns to the water at the same time as the body twists. The breathing made at the base of the bow, made by heading forward, with the head, is effective because it keeps the head in the shaft and thus reduces the specific resistance"'[4].

\section{Training program}

For lower limbs

- Squats with dumbbell at the back: 2 sets, 6 to 8 repetitions, 50 seconds break between sets;

- Squats with 2-3 $\mathrm{kg}$ dumbbells, from stand out: 3 sets, 6 to 8 repetitions, 50 break seconds between sets;

- Legs flexions from the position of lying face up: 3 sets, 6 to 8 repetitions, 45 seconds break between sets;

- Tip toes elevations on the gym equipment: 2 sets, 6 to 8 repetitions, 45 seconds break between the sets;

- 2-3 kg dumbbells lunges: 2 sets, 6 to 8 repetitions, 50 seconds break between sets;

- Double Dutch: 3 sets, 30 repetitions, break 50 seconds between sets.

- Squats with a dumbbell at the neck: 4 sets, 10-12 repetitions, 1 minute break between the sets;

- Dumbbell lunges: 4 sets, 6 to 8 repetitions 1 minute pause;
- Legs flexions from the position of lying face up: 4 sets, 10 to 12 repetitions, 50 seconds break between sets;

- Tip toes elevations on the gym equipment: 4 sets, $10-12$ repetitions, 1 minute break between the sets;

- Rounded shoulder twists on the bench: 4 sets, 10 to 12 repetitions, 50 seconds break between the sets;

- Side bending with dumbbells in standing position: 4 sets, 10-12 repetitions, 1 minute break between the sets.

- Squats with dumbbell at the back: 2 sets, 8 to 10 repetitions, 45 seconds break between the sets;

- Deep support lunges with dumbbells (one box was used as support): 4 sets, 8 to 10 repetitions, 50 seconds break between sets;

- Extensions of the leg from sitting to the gym apparatus: 4 sets, 8 to 10 repetitions, 45 seconds break between the sets;

- Hands to back lunges: 3 sets, 8 to 10 repetitions, 45 seconds break between the sets;

- Twisting lateral torsion of the trunk with a bar on the shoulders, standing on the bench: 4 sets, 8 to 10 repetitions;

- Side bending with dumbbells in the hands in the sitting position: 4 sets, 8 to 10 repetitions.

For the upper limbs, a circuit composed of three TRX exercise sequences, respectively, traction, rowing, simultaneously pushed from the legs, biceps pushups, extension and flexion of the arms for triceps, lifting with arms extended towards the upper limbs forward and up, as well as twisting, stretching, tilting, and extensions with difficulty.

The first sequence consists of:

- Close ups: 2 sets, 6 to 8 repetitions, 1 minute break between the sets;

- Biceps arm flexion: 2 sets, 6 to 8 repetitions, 45 seconds break between sets;

- Flexibility and extension of triceps arms: 2 sets, 6 to 8 repetitions, 45 seconds break between sets; 
- Shoulder elevations upwards for shoulders: 2 sets, 6 to 8 repetitions, break 50 seconds between evenings;

- Flops from palm rest to the ground: 2 sets, 6 to 8 repetitions, 1 minute break between the evening;

- Extension of the upper train maintaining 25-30 seconds

- The second sequence contains:

- Close-ups: 4 sets, 10 to 12 repetitions 1 minute break between sets;

- The flexion of the forearm on the biceps arm from a long distance: 2 sets, 10-12 repetitions, 45 seconds break between the sets;

- Flexibility and extension of the triceps arms, from standing apart: 2 sets, 10-12 repetitions, 45 seconds break between the sets;

- Shoulder elevations upwards for shoulders: 2 sets, 10 to 12 repetitions, break 50 seconds between sets;

- Twist with the shoulder bar on the bench: 2 sets, 10-12 repetitions, 45 seconds break between the sets;

- Flops from the palm rest to the ground with a palm beating: 4 sets, 10-12 repetitions, 1 minute break between the evening.

- The last sequence consists of:

- Close-ups: 3 sets, 8 to 10 repetitions, 1 minute break between the evening;

- Flexion of the forearm on the biceps arm, from a long distance: 3 sets, 8 to 10 repetitions, 45 seconds break between the sets;

- Flexibility and extension of triceps arms, from stand out: 3 sets, 8 to 10 repetitions, break 45 seconds between sets;

- Shoulder elevations upwards for shoulders: 3 sets, 8 to 10 repetitions, 50 seconds break between sets;

- Twisting lateral torsion of the trunk with the shoulder bar on the bench: 4 sets, 6 to 8 repetitions, 45 seconds break between the sets;

- Extension of the upper train maintaining 25-30 seconds.

\section{Research results}

After presenting the experiment group training program I will present recorded and interpreted data. The data we needed to observe an improvement in the performance of the subjects who had undergone a more intense training program we collected once at the start of the study with an initial test and then at the end of the training with a final test.

Next I will present the results to the crawl process:

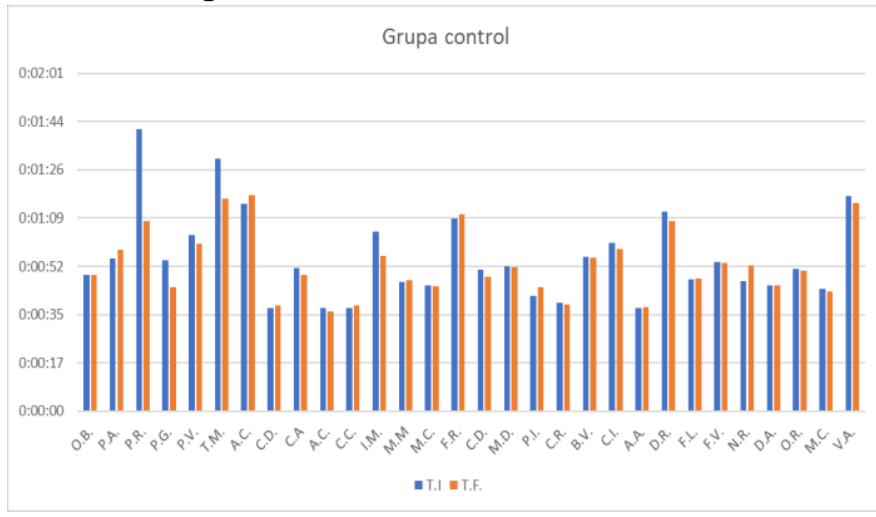

Figure no. 1 Comparative chart of initial times with final times in the control group for the crawl process 


\begin{tabular}{|l|r|r}
\hline & Variable 1 & Variable 2 \\
\hline Mean & 0,000636921 & 0,000610957 \\
\hline Variance & $3,33493 \mathrm{E}-08$ & $1,94294 \mathrm{E}-08$ \\
\hline Observations & 30 & 30 \\
\hline Hypothesized Mean Differ & 0 & \\
\hline df & 54 & \\
\hline t Stat & 0,619029451 & \\
\hline$P(T<=t)$ one-tail & 0,269249917 & \\
\hline t Critical one-tail & 1,673564906 & \\
\hline$P(T<=t)$ two-tail & 0,538499835 & \\
\hline t Critical two-tail & 2,004879288 & \\
\hline
\end{tabular}

Table no. 1 tTestCraulGC

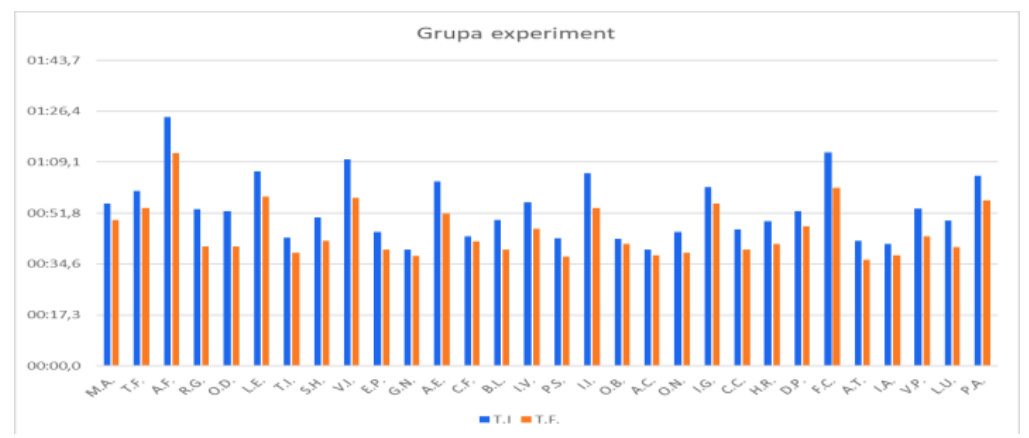

Figure no. 2 Comparative chart of initial times with GE final times for the crawl process

\begin{tabular}{|l|r|r|}
\hline \multicolumn{3}{|l|}{ t-Test: Two-Sample Assuming Unequal Variances } \\
\hline & Variable 1 & Variable 2 \\
\hline Mean & 0,000617052 & 0,000530054 \\
\hline Variance & $1,61511 \mathrm{E}-08$ & $1,06008 \mathrm{E}-08$ \\
\hline Observations & 30 & \\
\hline Hypothesized Mea & 0 & \\
\hline df & 56 & \\
\hline t Stat & 2,913362154 & \\
\hline P(T<=t) one-tail & 0,002564412 & \\
\hline t Critical one-tail & 1,672522303 & \\
\hline P(T<=t) two-tail & 0,005128824 & \\
\hline t Critical two-tail & 2,003240719 & \\
\hline
\end{tabular}

Table no.2 tTestCraulGE 


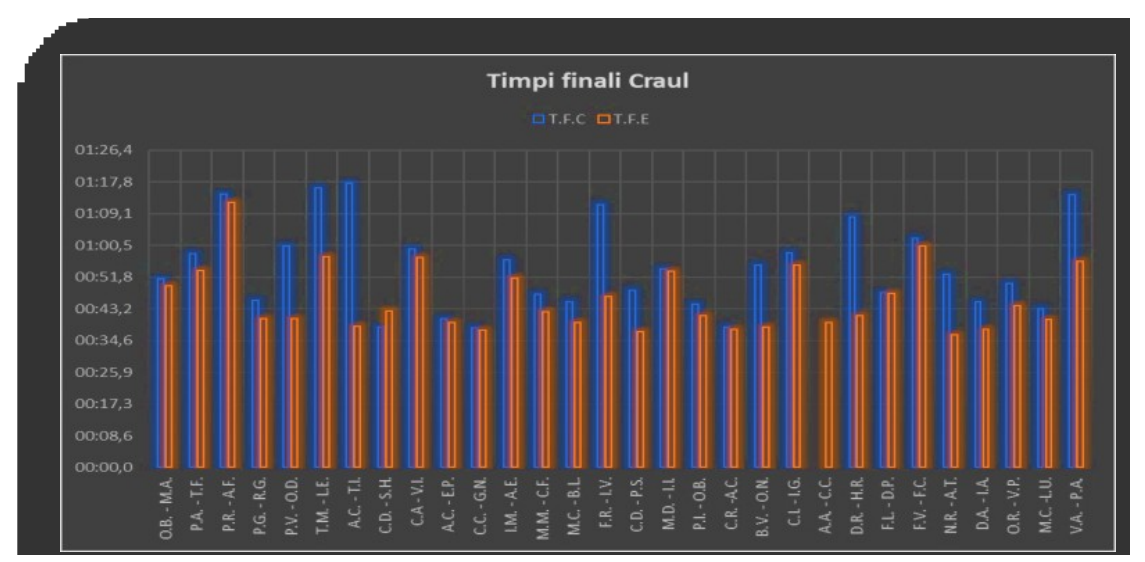

Figure no. 3Comparative chart between final control group and experimental procedures for the crawl process

\begin{tabular}{|l|r|r|}
\hline \multicolumn{3}{|c|}{$t$-Test: Two-Sample Assuming Unequal Varian } \\
\hline & & \\
\hline & Variable 1 & Variable 2 \\
\hline Mean & 0,000626 & 0,00053 \\
\hline Variance & $1,97 E-08$ & $1,06 E-08$ \\
\hline Observations & 30 & 30 \\
\hline Hypothesized Mean Diff & 0 & \\
\hline$d f$ & 53 & \\
\hline$t$ Stat & 3,018855 & \\
\hline$P(T<=t)$ one-tail & 0,001948 & \\
\hline$t$ Critical one-tail & 1,674116 & \\
\hline$P(T<=t)$ two-tail & 0,003896 & \\
\hline$t$ Critical two-tail & 2,005746 \\
\hline
\end{tabular}

Table no.3 Test Craul TFC-TFE

Using mathematical statistics, we performed a comparative analysis of the two groups (control and experiment) for the swimming process, as follows: In the case of the crawl process:

- the difference between initial testing and final testing within the control group is: $0.52 \mathrm{~s}$;

- the difference between initial testing and final testing within the experiment group is: $2.58 \mathrm{~s}$.

- the coefficient of variation, after the final test and implicitly after applying the training programs, is $22 \%$ for the control group and $20 \%$ for the experimental group, which means we have two equally homogeneous environments with a small plus for the group experiment.

- as we can see in the table above for a tst $=3.01>\mathrm{T}=1.67$ we can conclude that statistically we have a significant difference $\mathrm{p}(0.001)<0.05$ so there is a significant statistical difference.

\section{Research conclusions}

It can be seen with the graphs and the statistical data represented that the experimental group has a clear increase in 
the performance against the control group. With regard to the results obtained in the final tests, it can be noticed that again, the subjects of the experimental group managed to achieve better performances than those in the control group.

These performances were achievable due to the training program implemented for the experimental group that was followed with interest and maximum conscientiousness by the subjects of this group. Using the formulas we have used in Excel (arithmetic mean, minimum, maximum, Student test, etc.), a comparison can be made between the difference between the initial test and the final test.

Thus we can say that we have achieved the objectives we set at the beginning of the research, namely:

The students' availability to attend overtime has been noticed and identified to improve their water performance;

The ability and effort of military students to develop;

Some factors have also been identified in the preparation and development of subjects, such as the maintenance of a centralized training program.

The results of the students at the $50 \mathrm{~m}$ crack test are positively influenced by the adherence to the additional training program. The increase in performance is observed by decreasing the time of the 50m. crawl.

So we can conclude that additional physical training leads to improved performance.

\section{References}

[1] T.Laughlin, J.Delves, Total Immersion, The Revolutionary Way to Swim Better, Faster and Easier, Fireside, NY, USA, 2004, pp.10, 11

[2] J. Weineck, L'allenament. Ottimale, Calzetti Mariucci, 2001, p.66

[3] D. Ceontea, Inotul, Editura Napoca Star, Cluj-Napoca, 2008, p. 82.

[4] N.H. Pop, Curs Natație, Editura Napoca Star, Cluj-Napoca, 2010, pp. 44, 46, 48-49, 50 